\title{
Polyneuropathy, Organomegaly, Endocrinopathy, Monoclonal Gammopathy, and Skin Changes (POEMS) Syndrome With IgG Kappa/IgG Lambda Biclonal Gammopathy: A Rare Presentation of a Rare Disease
}

\author{
Leonardo Mejia Buritica $^{1}$, Vanessa Santiago-Pacheco ${ }^{2}$, Oliver Perilla ${ }^{1}$, Dayana Quintero ${ }^{3}$ \\ 1. Department of Hematology, Universidad de Antioquia, Medellín, COL 2. Department of Hematopathology, \\ Universidad de Antioquia, Medellín, COL 3. Department of Internal Medicine, Universidad Pontificia Bolivariana, \\ Medellín, COL
}

Corresponding author: Leonardo Mejia Buritica, leonardo.mejiab@udea.edu.co

\begin{abstract}
Polyneuropathy, organomegaly, endocrinopathy, monoclonal gammopathy, and skin changes (POEMS) syndrome is a low prevalence multisystemic paraneoplastic disease. The name is an acronym composed by its most relevant clinical manifestations, which are polyneuropathy, organomegaly, endocrinopathy, monoclonal gammopathy, and skin changes. More than $95 \%$ of the POEMS syndrome cases are monoclonal for lambda light chains; however, few cases have been reported in the literature with a biclonal component. In this paper, we report a rare case of a patient who has POEMS syndrome with biclonal gammopathy. To the best of our knowledge, this is the first reported case in the literature of POEMS syndrome with expression of IgG kappa/IgG lambda biclonal gammopathy.
\end{abstract}

Received 08/18/2020 Review began $08 / 22 / 2020$ Review ended 08/29/2020 Published 09/03/2020

๑) Copyright 2020

Mejia Buritica et al. This is an open access article distributed under the terms of the Creative Commons Attribution License CC-BY 4.0., which permits unrestricted use, distribution, and reproduction in any medium, provided the original author and source are credited.
Categories: Internal Medicine, Pathology, Hematology

Keywords: poems syndrome, biclonal gammopathy, castleman disease

\section{Introduction}

Polyneuropathy, organomegaly, endocrinopathy, monoclonal gammopathy, and skin changes (POEMS) syndrome is a low prevalence multisystemic paraneoplastic disease. The name is an acronym that highlights the most significant features: polyneuropathy, organomegaly, endocrinopathy, monoclonal gammopathy, and skin changes [1]. Other important manifestations of POEMS include fever, papilledema, volume overload, sclerotic bone lesions, thrombocytosis, erythrocytosis, and elevated levels of vascular endothelial growth factor (VEGF) [2]. Other names have previously been used to refer to the same clinical condition, such as Crow-Fukase syndrome, osteosclerotic myeloma, and Takatsuki syndrome [3]; however, the term POEMS introduced in 1980 is the most widely used nowadays [4].

It has been reported that between $11 \%$ and $30 \%$ of patients with POEMS syndrome have Castleman disease (giant lymph node hyperplasia) [5]; nevertheless, this percentage may be underestimated since not all patients undergo lymph node biopsy. A group of patients with Castleman disease without evidence of plasma cell neoplasm or neuropathy (and clinical characteristics of POEMS syndrome) has been described. These patients should be more accurately referred to as the Castleman disease variant of POEMS syndrome, since they may sometimes have a different clinical presentation from classic POEMS syndrome [1].

Regarding the monoclonal protein, more than $95 \%$ of the POEMS syndrome cases are monoclonal for lambda light chains [1]; however, few cases have been reported in the literature with a IgG kappa/IgA lambda biclonal component [6]. To the best of our knowledge, this article presents the first clinical case of a patient with POEMS syndrome with expression of IgG kappa/IgG lambda biclonal gammopathy.

\section{Case Presentation}

A 67-year-old woman with a history of hypertension and dyslipidemia presented with a five-month history of loss of strength in upper and lower extremities, progressive until bedridden. She also reported weight loss, fatigue, and generalized bone pain.

Physical examination revealed generalized hyperpigmentation and white nails, bilateral axillary lymphadenopathies of $3 \mathrm{~cm}$ in diameter, non-painful hepatomegaly, and pitting edema of the lower limbs. Neurological examination showed predominantly distal weakness, generalized areflexia, hypoesthesia of the legs, and inability to walk.

The complete blood count (CBC) revealed hemoglobin $16.5 \mathrm{~g} / \mathrm{dL}$, hematocrit $48.6 \%$, white blood cell count 


\section{Cureus}

10,200 cells $/ \mu \mathrm{L}$, and a normal platelet count $423,000 / \mu \mathrm{L}$. Endocrinological studies revealed mild hyperparathyroidism with parathyroid hormone (PTH) $72.9 \mathrm{pg} / \mathrm{mL}$ (reference range $15-68.3 \mathrm{pg} / \mathrm{mL}$ ), primary hypothyroidism with thyroid-stimulating hormone (TSH) $30.6 \mu \mathrm{IU} / \mathrm{mL}$ (reference range $0.55-4.78 \mu \mathrm{IU} / \mathrm{mL}$ ), and free thyroxine (FT4) $0.31 \mathrm{ng} / \mathrm{dL}$ (reference range 0.89-1.76 ng/dL). Liver and kidney tests were within the reference range. Studies for HIV, hepatitis B, hepatitis C, and syphilis were all negative.

Four-limb electromyography was performed, which showed demyelinating polyneuropathy of the lower extremities. Positron emission tomography CT (PET/CT) revealed hepatomegaly and polyostotic sclerotical bone lesions in both the right and the left humerus, the sternum, L3 vertebral body, the right acetabulum, the left sphenoid, the sacrum, and the trochanteric region of left femur (Figure 1).

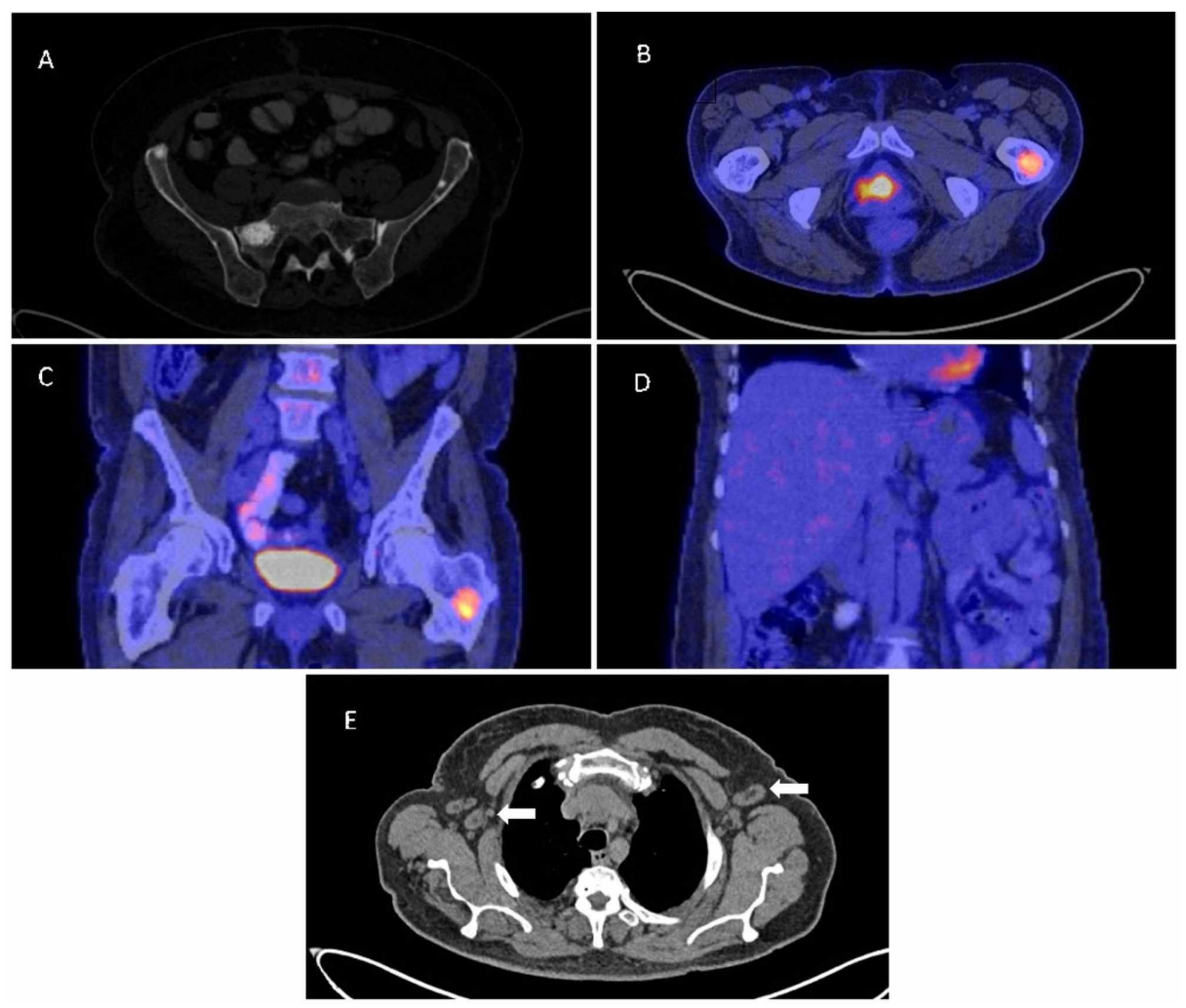

\section{FIGURE 1: Positron emission tomography CT (PET/CT) findings}

(A) Sclerotic lesions in the sacrum and iliac bone in cross section. (B) Sclerotic lesion with metabolic activity in the left femur in cross section. (C) Sclerotic lesion with metabolic activity in the left femur in coronal section. (D) Hepatomegaly in coronal section. (E) Bilateral axillary adenomegalies (arrows) in cross section.

Double peak in gamma was observed in serum protein electrophoresis and a biclonal component with IgG kappa/IgG lambda was demonstrated on immunofixation (Figure 2).

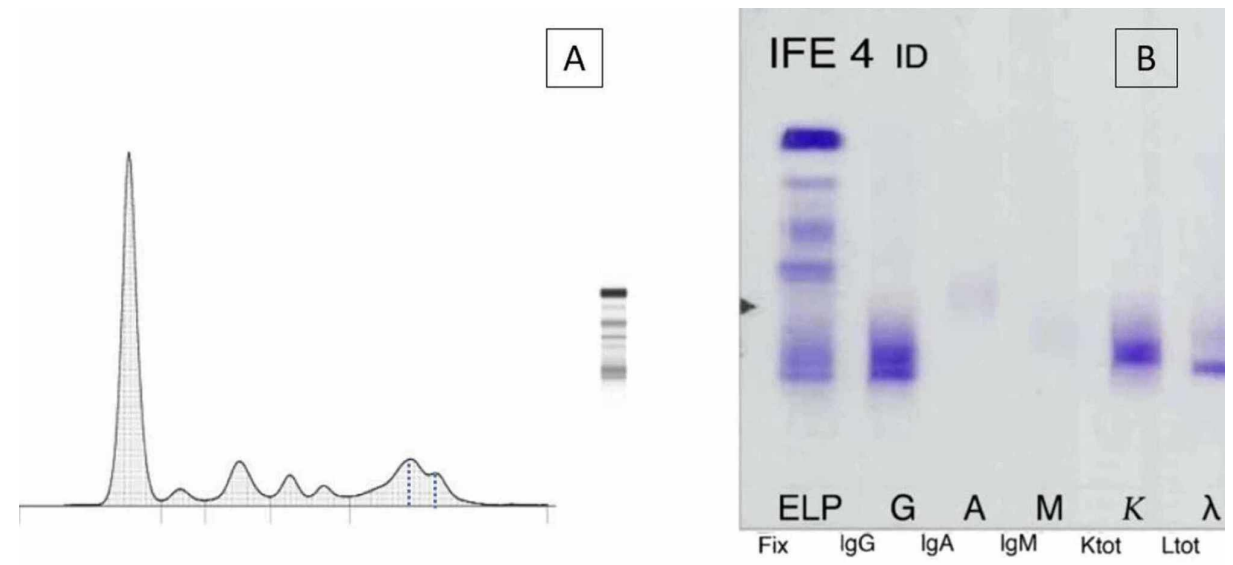

FIGURE 2: Serum electrophoresis and immunofixation 


\section{Cureus}

The light chain ratio was normal. Bone marrow biopsy revealed an increase in plasma cells with small aggregates, occupying approximately $10 \%$ of the medullary spaces with CD138 expression, with preserved kappa/lambda expression and aberrant expression of cyclin D1, which is associated with the 11;14 translocation. The biopsy of a cervical lymph node revealed morphological and immunophenotypic findings compatible with Castleman disease (Figure 3).
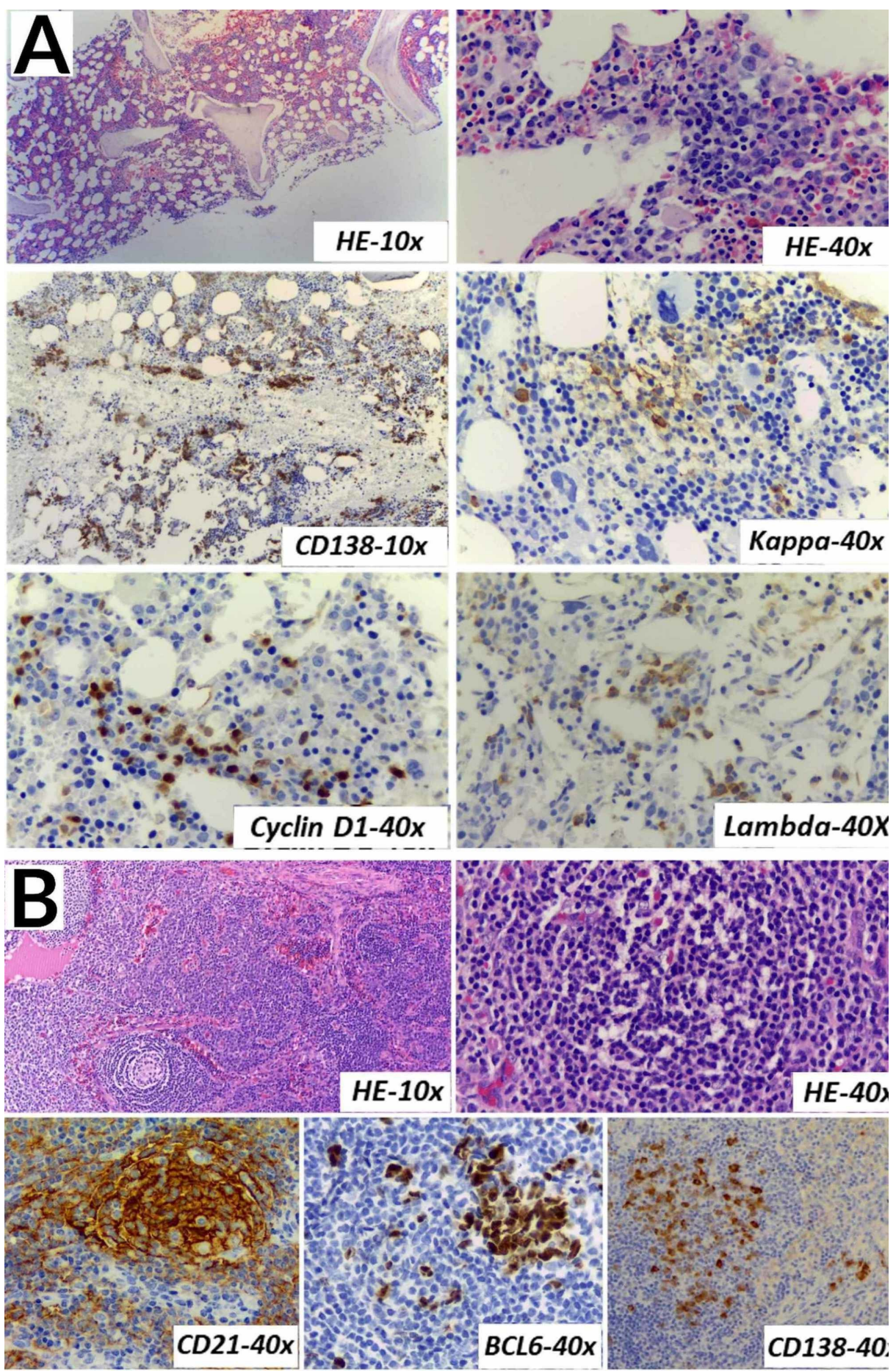

FIGURE 3: Bone marrow and cervical lymph node

(A) Bone marrow: It shows an increase in plasma cells with small aggregates, occupying approximately $10 \%$ of the medullary spaces (CD138), with preserved kappa/lambda expression and aberrant expression of cyclin D1. (B) Cervical lymph node: Lymphoid follicles with concentric hyperplasia of the mantle zone, proliferation of follicular dendritic cells (CD21), and small depleted germinal centers (BCL6) are observed. There is a low to moderate number of plasma cells (CD138). 
Treatment with lenalidomide and dexamethasone was started, with a rapid improvement in neurological symptoms.

\section{Discussion}

POEMS is a rare paraneoplastic clinical syndrome secondary to the presence of a plasma cell neoplasm [1]. The pathophysiology is not well understood, but it has been related to an increase in inflammatory cytokines, mainly VEGF [7]. Diagnostic criteria have been proposed by the International Myeloma Working Group [8].

Our case had biclonal component with IgG/kappa and IgG/lambda expression, which is rare in patients with POEMS, who usually have a lambda light chain monoclonal component in most cases [1]. Biclonal gammopathy is defined by the presence of two monoclonal components, which can be produced by the presence of two different plasma cell clones or by a single clone producing two monoclonal chains [6].

Biclonal gammopathy is rare and has been reported in $2.5 \%$ of cases of patients with monoclonal protein, most with a diagnosis of monoclonal gammopathy of undetermined significance in $65 \%$ of cases and multiple myeloma in $16 \%$ of cases [9]. Regarding the biclonal component, the most frequent finding is the combination of IgG and IgA observed in 53\% of cases, followed by the combination of IgM and IgG in $26 \%$ of cases [10]. Biclonal gammopathy with the combination of the IgG heavy chain with both monoclonal kappa and lambda light chains has been reported in non-Hodgkin lymphoma [11]. In POEMS syndrome, there are very few reported cases of biclonal gammopathy; the reports by De et al. [6] and Ham et al. [12] correspond to the combination of IgG and IgA. To our knowledge, this is the first reported case in the literature of POEMS syndrome with expression of IgG kappa/IgG lambda biclonal gammopathy.

Castleman disease, also known as angiofollicular lymph node hyperplasia, is a rare lymphoproliferative disease, which has been reported in up to $30 \%$ of patients with POEMS [5]. The classic POEMS syndrome must be differentiated from the Castleman disease variant of the POEMS syndrome [13]. In classic POEMS syndrome, there is evidence of plasma cell neoplasia and monoclonal component, with demyelinating peripheral neuropathy; the finding of Castleman disease in the lymph nodes may also be present. In contrast, in the Castleman disease variant of POEMS syndrome, there is no evidence of plasma cell neoplasia or monoclonal component and peripheral neuropathy is rare. In these cases, the presence of Castleman disease is necessary to establish the diagnosis [8]. Our case meets criteria for classic POEMS syndrome due to the presence of demyelinating polyneuropathy, monoclonal component, sclerotic bone lesions, enlarged lymph nodes, hepatomegaly, edema, endocrinopathy, and hyperpigmentation. The finding of Castleman's disease, in this case, additionally supports the diagnosis of POEMS syndrome.

The treatment of classic POEMS syndrome is usually directed at the plasma cell clone and depends mainly on whether there is a presence of solitary plasmacytoma or plasma cell infiltration in the bone marrow biopsy. For patients with isolated plasmacytoma without infiltration of plasma cells into the bone marrow, radiation therapy directed to plasmacytoma is the treatment of choice, which can resolve the clinical manifestations of the disease with an overall survival of $97 \%$ and failure-free survival of $52 \%$ at four years of follow-up [14].

Patients with two or more plasmacytomas or infiltration by plasma cells in the bone marrow can no longer be cured with radiotherapy and require systemic therapy, like that used in patients with multiple myeloma. Schemes combining bortezomib, cyclophosphamide, and dexamethasone (VRd) [15] or lenalidamide and dexamethasone (Rd) $[16,17]$ have been reported with good results.

In the case of young patients with multiple sclerotic lesions and severe clinical manifestations, high doses of melphalan chemotherapy followed by autologous stem cell transplantation should be considered. Significant improvement has been demonstrated with this therapy, especially in peripheral neuropathy [18].

\section{Conclusions}

POEMS syndrome is a rare disease associated with a plasma cell neoplasm. Its clinical manifestations are diverse, and the diagnosis is usually delayed. The classic form of the disease presents with demyelinating peripheral neuropathy and monoclonal gammopathy, usually lambda light chain. We present the case of a patient with IgG kappa/IgG lambda biclonal gammopathy associated with Castleman disease, who meets criteria for classic POEMS syndrome. Biclonal gammopathy is an exceedingly rare finding in POEMS syndrome. The importance of this finding regarding clinical evolution and response to treatment has not yet been established. With this case, we hope that the diagnosis of POEMS syndrome will also be considered in patients with this presentation. 


\section{Additional Information \\ Disclosures}

Human subjects: Consent was obtained by all participants in this study. Conflicts of interest: In compliance with the ICMJE uniform disclosure form, all authors declare the following: Payment/services info: All authors have declared that no financial support was received from any organization for the submitted work. Financial relationships: All authors have declared that they have no financial relationships at present or within the previous three years with any organizations that might have an interest in the submitted work. Other relationships: All authors have declared that there are no other relationships or activities that could appear to have influenced the submitted work.

\section{References}

1. Dispenzieri A: POEMS syndrome: 2019 Update on diagnosis, risk-stratification, and management . Am J Hematol. 2019, 94:812-827. 10.1002/ajh.25495

2. Kang J, Yang F, Zhang HY, et al.: POEMS syndrome associated with Castleman disease: a case report and literature review. Neuroimmunol Neuroinflamm. 2014, 1:40-43. 10.4103/2347-8659.135577

3. Nakanishi T, Sobue I, Toyokura Y, et al.: The Crow-Fukase syndrome: a study of 102 cases in Japan . Neurology. 1984, 34:712-720. 10.1212/wnl.34.6.712

4. Bardwick PA, Zvaifler NJ, Gill GN, Newman D, Greenway GD, Resnick DL: Plasma cell dyscrasia with polyneuropathy, organomegaly, endocrinopathy, M protein, and skin changes: the POEMS syndrome. Report on two cases and a review of the literature. Medicine. 1980, 59:311-322. 10.1097/00005792198007000-00006

5. Dispenzieri A, Kyle RA, Lacy MQ, et al.: POEMS syndrome: definitions and long-term outcome. Blood. 2003, 101:2496-2506. 10.1182/blood-2002-07-2299

6. De D, Halder S, Datta SS: POEMS syndrome with biclonal gammopathy: a rare association . J Clin Diagn Res. 2017, 11:XD12-XD13. 10.7860/JCDR/2017/24800.10265

7. D'Souza A, Hayman SR, Buadi F, et al.: The utility of plasma vascular endothelial growth factor levels in the diagnosis and follow-up of patients with POEMS syndrome. Blood. 2011, 118:4663-4665. 10.1182/blood2011-06-362392

8. Rajkumar SV, Dimopoulos MA, Palumbo A, et al.: International Myeloma Working Group updated criteria for the diagnosis of multiple myeloma. Lancet Oncol. 2014, 15:538-548. 10.1016/S1470-2045(14)70442-5

9. Kyle RA, Robinson RA, Katzmann JA: The clinical aspects of biclonal gammopathies. Review of 57 cases . Am J Med. 1981, 71:999-1008. 10.1016/0002-9343(81)90326-0

10. Sakashita C, Saito T, Kurosu T, et al.: Two M-components in a single cell lineage in a patient with a dual isotype secretory B-cell tumour. Br J Haematol. 1998, 102:791-794. 10.1046/j.1365-2141.1998.00832.x

11. Guarner J, Austin GE, Nassar VH, Sarma PR, Boedecker E: Biclonal gammopathy (IgG kappa and IgG lambda) in a patient with non-Hodgkin's lymphoma. Arch Pathol Lab Med. 1986, 110:445-448.

12. Ham JY, Suh JS, Lee WK, Song KE: POEMS syndrome with IgG- $\lambda$ /IgA-K biclonal gammopathy and abnormal serum free light chain ratio: A case report. Ann Clin Lab Sci. 2015, 45:702-706.

13. Yu H, Yao F, Li Y, Li J, Cui QC: Castleman disease variant of POEMS syndrome complicated with multiple cerebral infarction: A rare case report and review of literature. Int J Clin Exp Pathol. 2015, 8:13578-13583.

14. Humeniuk MS, Gertz MA, Lacy MQ, et al.: Outcomes of patients with POEMS syndrome treated initially with radiation. Blood. 2013, 122:68-73. 10.1182/blood-2013-03-487025

15. He H, Fu W, Du J, Jiang H, Hou J: Successful treatment of newly diagnosed POEMS syndrome with reduceddose bortezomib based regimen. Br J Haematol. 2018, 181:126-128. 10.1111/bjh.14497

16. Nozza A, Terenghi F, Gallia F, et al.: Lenalidomide and dexamethasone in patients with POEMS syndrome: results of a prospective, open-label trial. Br J Haematol. 2017, 179:748-755. 10.1111/bjh.14966

17. Li J, Huang XF, Cai QQ , et al.: A prospective phase II study of low dose lenalidomide plus dexamethasone in patients with newly diagnosed polyneuropathy, organomegaly, endocrinopathy, monoclonal gammopathy, and skin changes (POEMS) syndrome. Am J Hematol. 2018, 93:803-809. 10.1002/ajh.25100

18. D’Souza A, Lacy M, Gertz M, et al.: Long-term outcomes after autologous stem cell transplantation for patients with POEMS syndrome (osteosclerotic myeloma): a single-center experience. Blood. 2012, 120:5662. 10.1182/blood-2012-04-423178 